\title{
PICOSECOND-RESOLUTION 'SLICE' EMITTANCE MEASUREMENT OF ELECTRON-BUNCHES*
}

\author{
Ilan Ben-Zvi, Joe X. Qiu and Xijie Wang, National Synchrotron Light Source 725C, \\ Brookhaven National Laboratory, Upton NY 11973-500 USA
}

\section{Abstract}

The Slice Emittance diagnostic is applicable to particle bunches in a linac that are of the order of a few degrees of phase long. In this technique, the transverse phase space of a longitudinal slice about one degree long is measured. The Slice Emittance diagnostic has been demonstrated on an electron bunch produced by a laser-photocathode RF gun. We measured the transverse beam matrix of one picosecond slices out of a 10 picosecond long bunch (about 10 degrees at the RF frequency of $2856 \mathrm{MHz}$ ).

To implement this diagnostic one needs a phase shifter on part of the linac, a momentum analyzer (a dipole magnet followed by a slit) and a transverse emittance measuring system following the analyzer. By dephasing the last section (or sections) of the linac, longitudinal position in the bunch is correlated with energy. The momentum analyzer selects a short longitudinal slice by discriminating on energy and the transverse phase space of this slice is measured downstream of the analyzer.

The Slice Emittance diagnostic, particularly in conjunction with tomographic analysis of the transverse phase space of the slices, provides significant new information about the 6$D$ phase space distribution of the beam. The experimental work done with this diagnostic has been used to study the details of the important emittance compensation method that is in wide use in photoinjectors. This diagnostic makes it possible to take the emittance compensation method a step further and apply non-linear emittance compensation for higher brightness beams.

\section{THE SLICE EMITTANCE TECHNIQUE}

High brightness particle beams are an important frontier in accelerator physics. Our ability to measure beams in the greatest detail possible is the key for future progress. There is need for diagnostics to study in detail the phase space of the beam and provide guidance for multiparameter adjustment of the beam source and transport. The 6-D phase space distribution of a short longitudinal slice of a beam of electrons or other particles is particularly important. The slice-emittance technique [1] is such a diagnostic. In this technique, a short slice is selected out of an energy chirped beam by a slit in a dispersive region. The emittance of this slice is measured downstream of the slit using the quadrupole scan technique (or any other method). The measurement is repeated for different slices and for different beam conditions, like a new value for the current in the emittance compensating solenoid. We can also apply tomographic analysis techniques to the data taken in the quadrupole scan. Then we extract the density distribution in the transverse phase space slice by slice. This procedure provides us with a nearly complete characterization of the density distribution in the 6-D phase space of the beam.

\subsection{Description of the set-up and the measurement method}

Figure 1 shows the schematic layout of the sliceemittance measurement diagnostic at the BNL Accelerator Test Facility. The beam is generated at the photocathode by a $10 \mathrm{ps}$ long UV laser pulse. The electron bunch, in the measurement described below, has a charge of $0.4 \mathrm{nC}$. The gun output energy is $4 \mathrm{MeV}$ (corresponding to a 89 $\mathrm{MV} / \mathrm{m}$ peak electrical field on the cathode). The beam emerging from the gun is focused by a solenoid magnet and injected into a $2856 \mathrm{MHz}$ linear accelerator (linac). A laminar-flow beam waist is formed in the linac, during acceleration. The acceleration continues until the space charge forces become negligibly small, freezing the resultant phase space distribution.

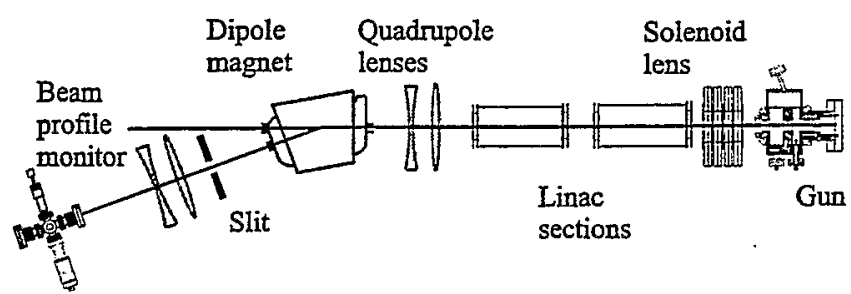

Figure: 1 Setup for the measurement of slice emittance at the BNL Accelerator Test Facility.

The linac consists of two sections. The phase of the second section is controlled independently by a motor controlled phase shifter. The high energy beam is bent horizontally by

* Work supported by the US Department of Energy under contract No. DE-AC02-76CH00016. 
a 20 degrees dipole magnet and a small slice in energy is selected by a slit. A quadrupole magnet following the slit is scanned in current and the vertical beam size is measured on a Beam Profile Monitor (BPM). The BPM comprises a phosphorous screen to form a luminous image of the beam intensity profile and a CCD camera to record it. The distance from the quadrupole to the BPM is $8.96 \mathrm{~m}$. For clarity we omit from the figure various beam transport quadrupole lenses in the high energy beam line.

The second linac section, dipole and slit form a filter that passes only a short slice of the beam pulse downstream of the slit. The quadrupole lenses and beam profile monitor downstream of the filter form an analyzer to measure the beam matrix of the slice. The filter is tuned to a given slice by changing the phase of the second linac section. Since the dipole current is constant, the energy of a selected slice is constant regardless of its position along the bunch, and the optics of the selected slices in the filter (and before it) are identical. However, under regular operation of the machine the slices enter the second linac section at slightly different phases around the crest of the accelerating wave-form. Thus they will experience a slightly different focusing. The maximum relative defocusing kick along a $10 \mathrm{ps}$ long bunch positioned at the crest is of the order of half a microradian. This is two orders of magnitude smaller than the angular spread in the approximately one millimeter diameter beam as it enters the second linac section and thus negligible.

The horizontal spot size on the slit is determined by the beta function at that point and by the energy spread.. The measured horizontal spot size was equivalent to an energy spread of $0.5 \%$ FWHM with the known $5.3 \mathrm{~mm} / \%$ dispersion at the slit.. The slit opening was adjusted to an opening of $2.6 \mathrm{~mm}$ equivalent to an energy bite of about $0.5 \%$. Next, the second linac section was dephased by 29.8 degrees to produce a nearly linear energy chirp of $0.439 \%$ per picosecond. Due to this chirp, the slit opening corresponded to $1.1 \mathrm{ps}$.

The experimental data was obtained by the BPM. The image of the beam is captured by a CCD camera ( Pulnix model 745E) with a frame grabber (Spiricon model LBA$100 A$ ). The CCD camera has pixel size of 11 by 13 microns and the number of pixels is 512 by 480 . A remotely controlled iris is used to avoid saturation in the 8 bit dynamic range of the frame grabber. The $\mathrm{Gd}_{2} \mathrm{O}_{2} \mathrm{~S}: \mathrm{Tb}$ phosphor is linear in light per charge output under our beam energy and charge conditions. The spatial resolution of the light emanating from the phosphor is better than 100 microns, thus our resolution is determined by a combination of the pixel size and the phosphor resolution.
This resolution is the basis for the error estimate in the emittance measurements. The digitized image from the frame grabber was written onto a diskette to be analyzed off-line. The analysis consists of integrating over the horizontal direction and obtaining the rms beam size for the vertical direction. Each vertical rms size corresponds to one point in a quadrupole scan. The beam size as a function of the quadrupole strength is used in a best-fit analysis to extract the beam matrix just upstream of the quadrupole. In this analysis we make the usual assumption that the transverse phase space distribution is an ellipse.

\subsection{Results of the ATF slice emittance measurement}

One application of this powerful technique is a study of emittance compensation in electron photoinjectors.. In the emittance compensation technique, suggested by Bruce Carlsten [2], the observed emittance growth due to linear components of space charge forces in the photoinjector is compensated by passing the electron beam through a laminar-flow beam-waist. The space charge interaction in this beam waist results in a differential rotation of the slice ellipses to bring them into alignment at some point downstream of this waist. To understand this emittance growth and compensation, one has to look at the slice emittance of a number of slices as they evolve. To go beyond this correction that is characterized by a single variable (the solenoid current), it is necessary to monitor the slice by slice emittance of the beam and deduce a nonlinear (multi-parameter) correction.

The beam matrix provides us with the emittance of the measured slice and the orientation of the phase-space ellipse. The measured normalized rms emittances of the three slices are $3.5 \pm 1.1,2.8 \pm 1.1$ and $2.3 \pm 1.1$ for the front, center and end of the bunch slices, respectively.

To illustrate the relative orientation between the slice ellipses, they were rotated with a known transfer matrix so that the phase space ellipse of the end slice is an erect ellipse and has a fixed beam size in each of the plots. Figure 2 shows only the relative rotation of the slice ellipses and a reversal in this differential rotation at some solenoid field does not imply an absolute reversal. The best emittance compensation is achieved when all the slice ellipses line up. This will reduce the emittance of the whole electron bunch by producing the smallest projected area, with summation weighted by the charge in each slice.

As the beam passes through the waist created by the solenoid, the ellipses of the slices rotate relative to each other since the space-charge force is not uniform along the bunch. According to Carlsten's theory, the angle of rotation is a function of the solenoid field and the charge 
distribution. We name the slices after their relative position in the electron bunch: front, middle and end. The front slice has the largest charge, the middle the second largest and the end the smallest relative charge.
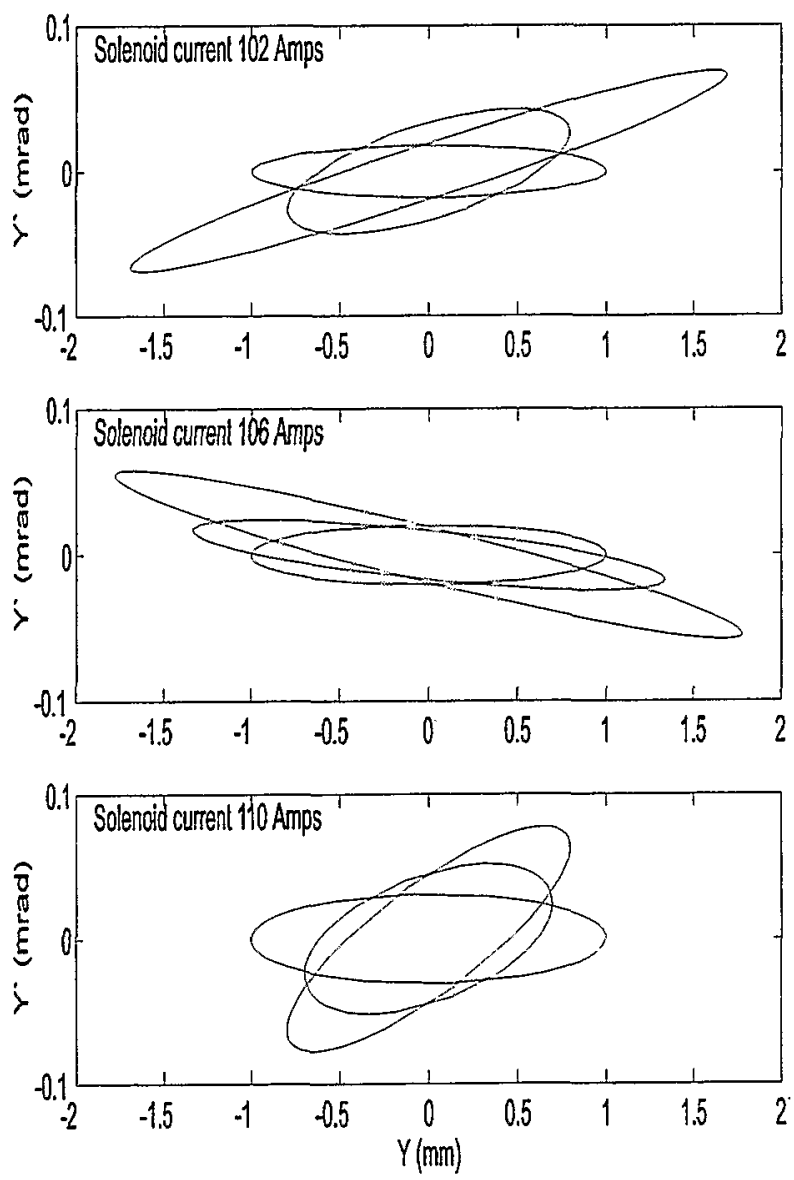

Figure: 2 The fitted beam ellipses for the slice emittance measurement for three solenoid currents. Top: $102 \mathrm{~A}$. Center: 106 A. Bottom: 110 A.The slice at the end of the beam bunch is taken as a beam waist, showing the relative rotation of the front and middle slices as the solenoid current is increased.

Comparing Figs. 2-top and 2-center we notice that the larger charge slices rotates through the larger angle, as expected. While Fig. 2-top (at $102 \mathrm{~A}$ ) represents an underrotation, Fig. 2-center. (at $106 \mathrm{~A}$ ) represents an overrotation (a point of near alignment was passed). As we continue to increase the solenoid current to 110 ampere, seen in Fig. 2-bottom., the relative rotation reverses itself, passing through near alignment once again. We expect that the closest alignment of the slice ellipses is attained at two solenoid currents, one between 102 and $106 \mathrm{~A}$, the other between 106 and $110 \mathrm{~A}$.

\section{THE FORMATION OF BEAM HALOS IN EMITTANCE COMPENSATION}

Emittance measurement, in particular that of a very small emittance, is difficult. One problem is the handling of beam halo. The process of emittance compensation can lead to a formation of a beam halo. The Carlsten model of emittance compensation uses a constant space charge force and thus predicts that compensation will apply to all longitudinal slices regardless of their current. A more detailed model, based on the envelope equation, shows that slices with a local current larger or smaller that some critical values will not fall into the compensation range and will form a beam halo. This will increase the integrated rms emittance, but due to the limited dynamic range of the frame grabber the measured value may be smaller since a diffuse halo may be missed.

As an example, consider the quad scan measurement of the total emittance as a function of solenoid current. As seen in Figure 3, the emittance initially decreases as expected as a result of emittance compensation. However, the increase in emittance is not as rapid as one would expect. This is the result of a cross over (non-laminar) of some part of the beam. A cross over results a large divergence and the creation of a low intensity beam halo that is not registered by a low dynamic range camera and frame-grabber system. The loss of the halo electrons due to the dynamic range results in a better emittance than should be observed otherwise.

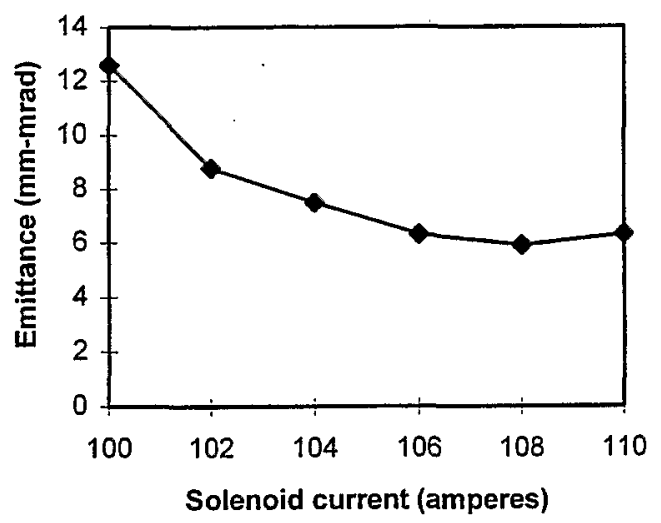

Figure: 3 Normalized total vertical emittance measured as a function of solenoid current.

The reason for the cross-over on some low current slices at a large solenoid field and the resultant beam halo can be shown by integrating the envelope equation of the beam in the linac. The relativistic envelope equation of a beam with space charge and emittance is given by: 
$R^{\prime \prime}+R^{\prime} \frac{\gamma^{\prime}}{\gamma}=\frac{2 I}{\gamma^{3} I_{A}} \frac{1}{R}+\frac{\varepsilon_{n}^{2}}{\gamma^{2}} \frac{1}{R^{3}}$

$R$ is the local envelope, $\gamma$ is the energy, $I_{A}$ is the van Alfven current, $I$ is the local current and $\varepsilon_{\mathrm{N}}$ is the normalized local emittance.

The emittance correction relies on a laminar flow beam waist, which is described by the envelope equation without the emittance term. We can define a critical beam waist envelope size, $R_{c}$ by equating the two terms on the right hand side, resulting in:

$$
R_{c}=\sqrt{\frac{\gamma \varepsilon_{n}{ }^{2} I_{A}}{2 I}}
$$

A beam waist that is larger (smaller) than the critical size, $R_{C}$, is dominated by space charge (emittance). The emittance dominated beam waist leads to a cross over of electrons at that particular slice. Since $R_{C}$ is a function of the current, the low current slices will cross over while high current ones will maintain a laminar flow.

This is not the problem with beam slices that have too little (or too much) current. The slope of the beam ellipse in phase space, given by $R^{\prime} / R$, can be tracked by integrating the envelope equation without the emittance term [3]. Plotting the slope at a point downstream of the solenoid lens as a function of beam current and various focusing powers of the lens, one learns the following:

- There are a few values of the focusing strength that result compensated beams.

- A lower limit and an upper limit slice current exist for each solution such that the slices with a current lower than the lower limit or higher than the upper limit will not align their slope with the compensated slices.

- The range of current for which the slope is constant (an emittance compensated range) depends on various parameters: initial conditions, position of the lens, position of the observation point, position and gradient of the accelerator (if there is one) and so on.

- The best solution is the first one (the lower lens power).

A Gaussian photocathode laser longitudinal distribution is thus bad for emittance compensation since it will result low current slices at the head and the tail of the electron bunch. The laser distribution may be modified on a sub-picosecond scale by known optical techniques. Using a slice-emittance measurement one may observe the results of the laser profile modification and tune the beam for a better emittance.

\section{TOMOGRAPHIC MEASUREMENT OF SLICE EMITTANCE}

By discussing beam ellipses or by making a-priory assumptions concerning the distribution of the beam in phase space in order to fit a quadrupole scan data (or other measurement techniques) to some beam parameters we are loosing a lot of information. As has been shown by McKee, O'Shea and Madey [4], one can apply tomographic techniques to a quadrupole scan and derive the full phase space distribution. This is a powerful technique that, together with the slice emittance measurement technique can provide a greater understanding of the beams of photoinjectors and help improve the performance.

Figure 4 shows the measured phase space density distribution of the total beam (not just one slice). The horizontal axis is a transverse coordinate (horizontal, in this case) in $\mathrm{mm}$, and the vertical axis is the divergence in mrad. This is an emittance compensated beam from the ATF photoinjector. It is possible to see the typical beam halo that shows up in measurements even with the limitted dynamic range ( 8 bits) of our frame grabber. The measured quadrupole-scan rms normalized emittance is about $4.5 \mathrm{~mm}$ mrad. However, as discussed above, the large halo causes an underestimation of the emittance.

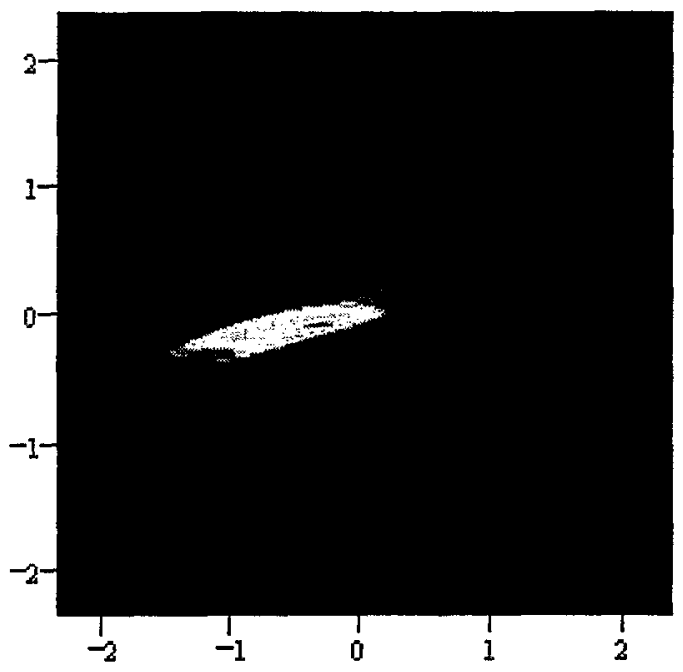

Figure: 4 Tomographic reconstruction of the horizontal non-normalized phase space of the total bunch of an emittance compensated beam. The horizontal axis is in milimeters, the vertical axis is in miliradians.

Figures 5,6 and 7 show the (transverse-vertical) phase space distribution of a beam slice of 50,100 and $200 \mathrm{pC}$, respectively. (Please notice the change of scale from Figure 4 , but the units are still $\mathrm{mm}$ and mrad). Some of the 
'splatter' in figures 6 and, in particular 7, is due to the fact that the measurement was not designed originaly as a tomographic measurement but as a plain quadrupole scan emittance measurement. Nevertheless, the significant features of the distribution are clear enough. The measured quadrupole-scan rms normalized emittances are 2.5, 2.9

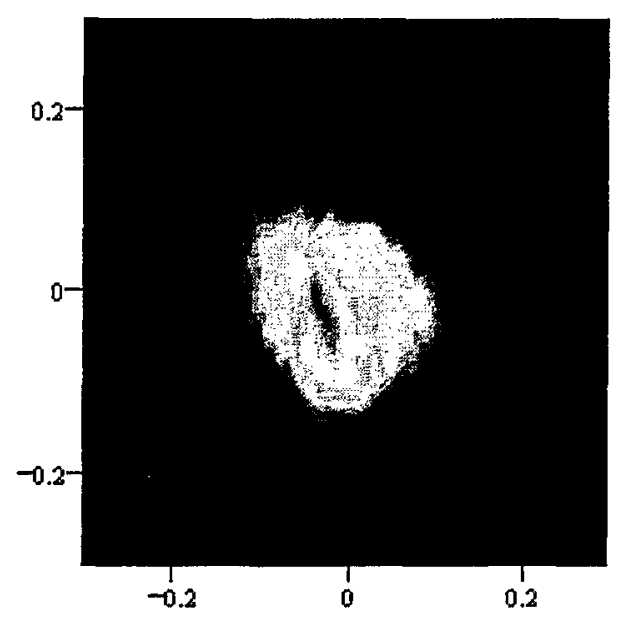

Figure: 5 Tomographic reconstruction of the vertical nonnormalized phase space of a slice. The horizontal axis is in $\mathrm{mm}$, the vertical axis is in mrad. The total charge is $50 \mathrm{pC}$.

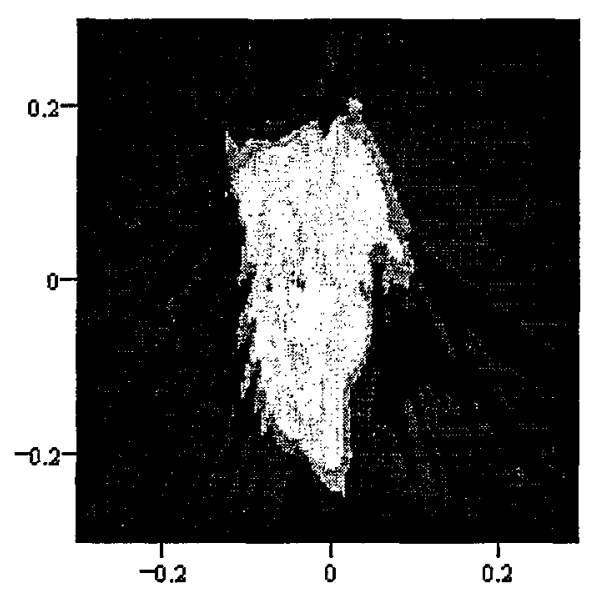

Figure: 6 Tomographic reconstruction of the vertical nonnormalized phase space of a slice. The horizontal axis is in $\mathrm{mm}$, the vertical axis is in mrad. The total charge is $100 \mathrm{pC}$.

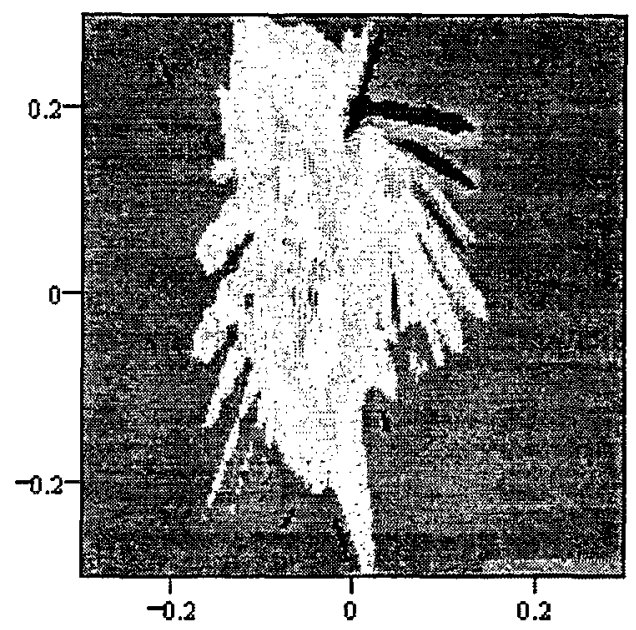

Figure: 7 Tomographic reconstruction of the vertical nonnormalized phase space of a slice. The horizontal axis is in $\mathrm{mm}$, the vertical axis is in mrad. The total charge is $200 \mathrm{pC}$.

and $3.7 \mathrm{~mm}$ mrad, respectively. Most of the halo seen in the full beam measurement is gone, since it is formed by low current slices that are cut out by the slice-emittance filter. As a result the emittance measured by a quadrupole scan technique is more believable. As we increase the charge, we see that most of the emittance increase is through spreading of the core and some distortion. This is a new information that may be used now for a better understanding of the emittance growth mechanism and, hopefully, to improvement of the brightness of the beam.

\section{REFERENCES}

[1] 'Demonstration of emittance compensation through the measurement of the slice emittance of a 10 picosecond electron bunch', X. Qiu, K. Batchelor, I. Ben-Zvi and X.J. Wang, Phys. Rev. Let. 76 No. 20, 3723, (1996)

[2] 'New Photoelectric Injector Design for the LANL XUV FEL Accelerator', B.E. Carlsten, Nucl. Instr. And Meth. In Phys. Res. A285, 313 (1989)

[3] Ph.D. dissertation, J.X. Qiu, The University at Stony Brook, ny 1997 (unpublished)

[4] 'Phase Space Tomography of Relativistic Electron beams', C.B. McKee, P.G. O'Shea and J.M.J. Madey, Nucl. Inst. And Meth. In Phys. Res. A358, 264 (1995). 